\title{
LIPSCHITZ MEASURES AND VECTOR-VALUED HARDY SPACES
}

\author{
MAGALI FOLCH-GABAYET, MARTHA GUZMÁN-PARTIDA, \\ and SALVADOR PÉREZ-ESTEVA
}

(Received 24 January 2000)

\begin{abstract}
We define certain spaces of Banach-valued measures called Lipschitz measures. When the Banach space is a dual space $X^{*}$, these spaces can be identified with the duals of the atomic vector-valued Hardy spaces $H_{X}^{p}\left(\mathbb{R}^{n}\right), 0<p<1$. We also prove that all these measures have Lipschitz densities. This implies that for every real Banach space $X$ and $0<p<1$, the dual $H_{X}^{p}\left(\mathbb{R}^{n}\right)^{*}$ can be identified with a space of Lipschitz functions with values in $X^{*}$.
\end{abstract}

2000 Mathematics Subject Classification. Primary 42B30, 46E30.

1. Introduction and notation. An interesting question in the theory of vectorvalued Hardy spaces is the representation of their dual spaces. This matter has been considered by several authors in the different versions (not necessarily equivalent) of these spaces, namely the $H^{p}$-spaces of holomorphic functions in the disk with values in a Banach space $X$ as well as the "maximal" and the atomic Hardy spaces of Banachvalued distributions in the unit circle (cf. [1, 2, 3, 4, 5]). In particular, in [1], it is proved that for every Banach space $X$, the dual of the atomic space $H_{\mathrm{at}}^{1}(X)$ in the unit circle is the space $\mathscr{B} M O\left(X^{*}\right)$ of measures in the circle and values in $X^{*}$ of bounded mean oscillation. In that paper, it is proved that this space of measures is in fact the space of functions of bounded mean oscillation if and only if $X^{*}$ has the Radon-Nikodym property and in this case $H_{\mathrm{at}}^{1}(X)^{*}=\mathscr{B} M O\left(X^{*}\right)$.

In this paper, we consider the atomic Hardy spaces $H_{X}^{p}$ in $\mathbb{R}^{n}$ for $0<p<1$. The dual space of each of these $H_{X}^{p}$ will be a space of measures with values in $X^{*}$ modulo polynomials of degree $N \leq[n(1 / p-1)]$ (measures with polynomial densities). These measures will be finitely additive measures defined in the ring of all bounded Borel sets in $\mathbb{R}^{n}$ and have a control imposed on cubes analogous to that one shared by the Lipschitz functions: $f \in L_{\text {loc }}^{1}\left(\mathbb{R}^{n}\right)$ is a Lipschitz function with exponent $\alpha>0$ if and only if there exists a positive constant $C>0$ such that

$$
\left\|f-P_{Q}^{[\alpha]}(f)\right\|_{L^{2}(Q)} \leq C|Q|^{(\alpha / n)+1 / 2},
$$

for every cube $Q$, where $P_{Q}^{[\alpha]}(f)$ is the unique polynomial of degree less than or equal to $[\alpha]$ having the same moments of order less than or equal to $[\alpha]$ as $f$.

The exponent $p \in(0,1)$ makes possible to adapt the arguments of the scalar theory to approximate these measures by measures with smooth density. The final result is that every measure belonging to our space of measures denoted by $\mu_{X}^{\alpha}$ has a RadonNikodým derivative that is a Lipschitz function with exponent $\alpha=n(1 / p-1)$. Then 
the dual of $H_{X}^{p}$ is the space of Lipschitz functions in $\mathbb{R}^{n}$ with values in $X^{*}$ modulo polynomials of degree less than or equal to $[\alpha]$. This holds for every real Banach space $X$, contrasting with the case $p=1$ mentioned above.

The following notation will be used throughout this paper, $\mathscr{Y}\left(\mathbb{R}^{n}, X\right)$ will denote the space of rapidly decreasing functions on $\mathbb{R}^{n}$ with values in a real Banach space $X$. We will denote by $\mathscr{D}^{\prime}\left(\mathbb{R}^{n}, X\right)$ the space of $X$-valued distributions in $\mathbb{R}^{n}$, that is, the space of all continuous linear operators from $C_{c}^{\infty}\left(\mathbb{R}^{n}\right)$ into $X$ and $\mathscr{S}^{\prime}\left(\mathbb{R}^{n}, X\right)$ will be the space of all the linear and continuous operators from $\mathscr{S}\left(\mathbb{R}^{n}\right)$ with values in $X . \mathscr{S}_{s}^{\prime}\left(\mathbb{R}^{n}, X\right)$ and $\mathscr{S}_{b}^{\prime}\left(\mathbb{R}^{n}, X\right)$ will denote, respectively, $\mathscr{S}^{\prime}\left(\mathbb{R}^{n}, X\right)$ with the topologies of pointwise convergence and uniform convergence in bounded subsets of $\mathscr{Y}\left(\mathbb{R}^{n}\right)$.

For a cube $Q \subset \mathbb{R}^{n}$ and $1 \leq p<\infty, L_{X}^{p}(Q)$ will denote the space of all the $X$-valued Bochner measurable functions on $Q$ such that $\|f\|$ belongs to $L^{p}(Q)$.

For $1 \leq q<\infty, V_{X}^{q}(Q)$ will be the space of all countably additive measures $\mu$ on the Borel sets on the cube $Q$ with values in $X$ and with finite $q$-variation

$$
\|\mu\|_{V_{X}^{q}(Q)}=\sup \left(\sum_{A \in \pi} \frac{\|\mu(A)\|^{q}}{m(A)^{q-1}}\right)^{1 / q},
$$

where the supremum is taken over all finite partitions (measurable) $\pi$ of $Q$ and $m$ is the Lebesgue measure. For $p>1$, the dual space of $L_{X}^{p}(Q)$ can be identified with $V_{X^{*}}^{q}(Q)$, where $1 / p+1 / q=1$. For complete expositions of vector measures and vector integration see $[6,7]$.

All the cubes considered here will be compact and will have sides parallel to the axes. $\sum_{0}$ will be the ring of bounded Borel sets in $\mathbb{R}^{n}$.

For $f \in \mathscr{Y}^{\prime}\left(\mathbb{R}^{n}, X\right)$ and $\varphi \in \mathscr{Y}\left(\mathbb{R}^{n}\right)$, we define

$$
M_{\varphi}^{*}(f)(x)=\sup _{|y-x|<t}\left\|\varphi_{t} * f(y)\right\|_{X} .
$$

$\Delta_{h} g(x)$ denote the difference $g(x+h)-g(x)$, where $g$ is an $X$-valued function and $\Delta_{h}^{2} g=\Delta_{h} \Delta_{h} g$. For $\alpha>0$, the spaces $\Lambda_{X}^{\alpha}$ will be the vector-valued versions of the spaces $\Lambda^{\alpha}$ of Lipschitz functions, namely, if $\alpha=[\alpha]+\alpha^{\prime}, 0<\alpha^{\prime}<1$, then $g \in \Lambda_{X}^{\alpha}$ if $g \in C_{X}^{[\alpha]}\left(\mathbb{R}^{n}\right)$ and

$$
\|g\|_{\Lambda_{X}^{\alpha}}=\sup _{|\beta|=[\alpha]} \sup _{h \in \mathbb{R}^{n} \backslash 0}|h|^{-\alpha^{\prime}}\left\|\Delta_{h} D^{\beta} \mathcal{g}\right\|_{L_{X}^{\infty}}<\infty
$$

For $\alpha \in \mathbb{Z}, g \in \Lambda_{X}^{\alpha}$ if $g \in C_{X}^{\alpha-1}\left(\mathbb{R}^{n}\right)$ and

$$
\|g\|_{\Lambda_{X}^{\alpha}}=\sup _{|\beta|=\alpha-1} \sup _{h \in \mathbb{R}^{n} \backslash 0}|h|^{-1}\left\|\Delta_{h}^{2} D^{\beta} g\right\|_{L_{X}^{\infty}}
$$

Notice that, as in the case of scalar functions, $\|g\|_{\Lambda_{X}^{\alpha}}=0$ implies that $D^{\beta} g$ is constant for all $|\beta|=[\alpha]$ if $\alpha \notin \mathbb{Z}$, and $D^{\beta} g$ is an affine linear function for every $|\beta|=\alpha-1$ when $\alpha \in \mathbb{Z}$. Using this fact and Taylor's theorem for Banach-valued functions, we conclude that $g$ is a polynomial of degree less than or equal to $[\alpha]$. 
We define $\widetilde{\Lambda_{X}^{\alpha}}$ to be the normed quotient space of $\Lambda_{X}^{\alpha}$ modulo polynomials of degree less than or equal to $[\alpha]$.

As usual, the letter $C$ denote a constant that could be different at each occurrence.

2. Interpolation polynomials and Lipschitz measures. The aim of this section is to define an appropriate space of vector-valued measures, which turn out to be the dual of a vector-valued Hardy space.

Let $Q$ be a cube on $\mathbb{R}^{n}$ with center $x_{0}$ and let $f \in L^{1}(Q)$. The linear independence of the family of functions $\left\{x^{\alpha}\right\}_{|\alpha| \leq N}$ implies the existence of a unique polynomial $P_{Q}^{N}$ of degree less than or equal to $N$ such that, for every multi-index $\alpha$ with $|\alpha| \leq N$,

$$
\int_{Q}\left(x-x_{0}\right)^{\alpha} f(x) \frac{d x}{|Q|}=\int_{Q}\left(x-x_{0}\right)^{\alpha} P_{Q}^{N}(x) \frac{d x}{|Q|} .
$$

This polynomial $P_{Q}^{N}$ can be constructed in the standard way using the dual basis of the set $\left\{\left(x-x_{0}\right)^{\alpha}:|\alpha| \leq N\right\}$ in $L^{2}(Q, d x /|Q|)$, that is, the set of polynomials $\left\{\psi_{\alpha}^{Q}\right\}_{|\alpha| \leq N}$ of degree less than or equal to $N$, such that

$$
\int_{Q}\left(x-x_{0}\right)^{\alpha} \psi_{\beta}^{Q}(x) \frac{d x}{|Q|}=\delta_{\alpha \beta},
$$

where $\delta_{\alpha \beta}$ is the Kronecker delta.

Then the interpolating polynomial is given by

$$
P_{Q}^{N} f(x)=\sum_{|\alpha| \leq N} a_{\alpha} \psi_{\alpha}^{Q}(x)
$$

where

$$
a_{\alpha}=\int_{Q} f(x)\left(x-x_{0}\right)^{\alpha} \frac{d x}{|Q|} .
$$

Let $Q_{1}$ be the cube centered at zero and side length 1 and take any cube $Q$ with center $x_{0}$ and side length $\delta$. If we let $\psi_{\alpha}=\psi_{\alpha}^{Q_{1}}$ for every $|\alpha| \leq N$, then

$$
\psi_{\alpha}^{Q}(x)=\delta^{-|\alpha|} \psi_{\alpha}\left(\frac{x-x_{0}}{\delta}\right) .
$$

Then we conclude that there exists a constant $C>0$ independent of $Q$ and $\alpha$, such that

$$
\left|\psi_{\alpha}^{Q}(x)\right| \leq C|Q|^{-|\alpha| / n}, \quad x \in Q .
$$

THEOREM 2.1. Given a cube $Q$ centered at $x_{0}, N \in \mathbb{N} \cup\{0\}, q \geq 1$ and $f \in L_{X}^{q}(Q)$, there exists a unique polynomial $P_{Q}^{N} f: \mathbb{R}^{n} \rightarrow X$ of degree less than or equal to $N$ such that

$$
\int_{Q} P_{Q}^{N} f(x)\left(x-x_{0}\right)^{\alpha} \frac{d x}{|Q|}=\int_{Q} f(x)\left(x-x_{0}\right)^{\alpha} \frac{d x}{|Q|}
$$

for every multi-index $\alpha$ with $|\alpha| \leq N$. This polynomial $P_{Q}^{N} f$ satisfies

$$
\left\|P_{Q}^{N} f(x)\right\|_{X} \leq \frac{C}{|Q|^{1 / q}}\|f\|_{L_{X}^{q}(Q)}, \quad x \in Q .
$$

Thus

$$
\left\|P_{Q}^{N} f\right\|_{L_{X}^{q}(Q)} \leq C\|f\|_{L_{X}^{q}(Q)} .
$$


Proof. Let $a_{\alpha}=\int_{Q} f(x)\left(x-x_{0}\right)^{\alpha} d x /|Q|$ and $P_{Q}^{N} f(x)=\sum_{|\alpha| \leq N} a_{\alpha} \psi_{\alpha}^{Q}(x)$. Then if $\beta$ is a multi-index with $|\beta| \leq N$, then

$$
\int_{Q} P_{Q}^{N} f(x)\left(x-x_{0}\right)^{\beta} \frac{d x}{|Q|}=a_{\beta}=\int_{Q} f(x)\left(x-x_{0}\right)^{\beta} \frac{d x}{|Q|} .
$$

To prove the uniqueness, suppose that $\int_{Q} P(x)\left(x-x_{0}\right)^{\alpha} d x /|Q|=0$ for every $\alpha$ with $|\alpha| \leq N$, where $P$ is a polynomial of degree less than or equal to $N$. Then, for every $\xi^{*} \in X^{*}$,

$$
\int_{Q} \xi^{*} \circ P(x)\left(x-x_{0}\right)^{\alpha} \frac{d x}{|Q|}=0
$$

and since $\xi^{*} \circ P$ is a polynomial with scalar coefficients whose degree is less than or equal to $N$, it follows that $\xi^{*} \circ P=0$ for every $\xi^{*} \in X$, therefore $P=0$. Finally, given $x \in Q$,

$$
\left\|P_{Q}^{N} f(x)\right\|_{X} \leq \sum_{|\alpha| \leq N}\left\|a_{\alpha}\right\|_{X}|Q|^{-|\alpha| / n}
$$

From Hölder's inequality, we have

$$
\left\|a_{\alpha}\right\|_{X} \leq C|Q|^{(|\alpha| / n)-1 / q}\left(\int_{Q}\|f(x)\|_{X}^{q} d x\right)^{1 / q}
$$

and this implies the desired estimates.

Let $Q$ be any cube centered at $x_{0}$ and $\mu \in V_{X}^{q}(Q)$. As in Theorem 2.1, we can construct

$$
P_{Q}^{N} \mu(x)=\sum_{|\alpha| \leq N} a_{\alpha} \psi_{\alpha}^{Q}(x)
$$

where $a_{\alpha}=1 /|Q| \int_{Q}\left(x-x_{0}\right)^{\alpha} d \mu$.

As in Theorem 2.1 we can prove the following corollary.

COROLlary 2.2. Let $\mu \in V_{X}^{q}(Q)$. Then $P_{Q}^{N} \mu$ is the unique polynomial of degree less than or equal to $N$ satisfying

$$
\int_{Q} P_{Q}^{N} f(x)\left(x-x_{0}\right)^{\alpha} \frac{d x}{|Q|}=\frac{1}{|Q|} \int_{Q}\left(x-x_{0}\right)^{\alpha} d \mu
$$

for every $|\alpha| \leq N$. The polynomial $P_{Q}^{N} \mu$ verifies

$$
\left\|P_{Q}^{N} \mu(x)\right\|_{X} \leq \frac{C}{|Q|^{1 / q}}\|\mu\|_{V_{X}^{q}(Q)}, \quad x \in Q, \quad\left\|P_{Q}^{N} \mu\right\|_{L_{X}^{q}(Q)} \leq C\|\mu\|_{V_{X}^{q}(Q)} .
$$

To abbreviate notation, we often write $P_{Q} f$ or $P_{Q} \mu$ if the context does not cause confusion. We introduce the following space of vector-valued measures. 
DEFinItION 2.3. Let $\mu: \sum_{0} \rightarrow X$ be a vector measure, $m$ the Lebesgue measure on $\sum_{0}, 0 \leq \alpha<\infty$. We say that $\mu \in \mathcal{M}_{X}^{\alpha}$, if the following conditions hold:

(i) $\mu \in V_{X}^{2}(Q)$, for every cube $Q$.

(ii) There exists a constant $C$ such that, for every cube $Q$,

$$
\left\|\mu-P_{Q}^{[\alpha]}(\mu) m\right\|_{V_{X}^{2}(Q)} \leq C|Q|^{(\alpha / n)+1 / 2} .
$$

For $\mu \in \mathcal{M}_{X}^{\alpha}$, we define

$$
\|\mu\|_{\mathcal{M}_{X}^{\alpha}}=\inf \{C: \text { (ii) holds }\} .
$$

$\|\cdot\|_{\mathcal{M}_{X}^{\alpha}}$ is a seminorm on $\mathcal{M}_{X}^{\alpha}$ and $\|\mu\|_{\mathcal{M}_{X}^{\alpha}}=0$ if and only if $\mu$ is a measure with polynomial density $P$ such that $\operatorname{deg} P \leq[\alpha]$. If we form the quotient space of $\mu_{X}^{\alpha}$ modulo polynomials of degree less than or equal to $[\alpha]$ (measures with polynomial density) we obtain a normed space $\widetilde{M_{X}^{\alpha}}$.

As in the scalar case, we have

$$
\|\mu\|_{\mathcal{M}_{X}^{\alpha}} \sim \sup _{Q}|Q|^{-\alpha / n} \inf _{\operatorname{deg} P \leq[\alpha]} \frac{1}{|Q|^{1 / 2}}\|\mu-P m\|_{V_{X}^{2}(Q)}
$$

From now on, we refer to this space as the space of Lipschitz measures $\mathcal{M}_{X}^{\alpha}$.

Every function $g \in \Lambda_{X}^{\alpha}$ defines a measure in $\mu_{X}^{\alpha}$, in fact, as in [9, Lemma 5.18, Chapter III], given $x_{0} \in \mathbb{R}^{n}$ and $r>0$, there exists a polynomial $P(x)$ of degree $[\alpha]$ such that

$$
\|g(x)-P(x)\|_{X} \leq C r^{\alpha}\|g\|_{\Lambda_{X}^{\alpha}}
$$

for every $x$ in the cube centered in $x_{0}$ and side length $r$, where $C$ only depends on $\alpha$ and $n$. This implies that

$$
\|g\|_{M_{X}^{\alpha}} \leq C\|g\|_{\Lambda_{X}^{\alpha}}
$$

where $\|g\|_{\mathcal{M}_{X}^{\alpha}}$ denotes the norm of the measure with density $g$. In Section 3, we prove that every $\mu \in \mathcal{M}_{X}^{\alpha}$ has a density $g \in \Lambda_{X}^{\alpha}$. Now, we can prove a weak version of this fact.

LEMMA 2.4. Let $\alpha>0$ and $g \in C_{X}^{m}\left(\mathbb{R}^{n}\right)$, where $m=[\alpha]$ if $\alpha$ is not an integer and $m=\alpha-1$ otherwise. If the measure with density $g$ belongs to $\mu_{X}^{\alpha}$, then $g \in \Lambda_{X}^{\alpha}$ and $\|g\|_{\Lambda_{X}^{\alpha}} \leq C\|g\|_{\mu_{X}^{\alpha}}$.

Proof. For every $\xi^{*} \in X^{*}$ and every cube $Q$, we have that $P_{Q}^{[\alpha]}\left(\xi^{*} \circ g\right)=$ $\xi^{*} \circ P_{Q}^{[\alpha]}(g)$. Then it is easy to see that $\xi^{*} \circ g \in \mathcal{M}_{\mathbb{R}}^{\alpha}$ and $\left\|\xi^{*} \circ g\right\|_{\mathcal{M}_{\mathbb{R}}^{\alpha}} \leq\left\|\xi^{*}\right\|_{X^{*}}\|g\|_{\mathcal{M}_{X}^{\alpha}}$. By the scalar theory we have that $\xi^{*} \circ g \in \Lambda^{\alpha}$ and

$$
\left\|\xi^{*} \circ g\right\|_{\Lambda^{\alpha}} \leq C\left\|\xi^{*} \circ g\right\|_{\mathcal{M}_{\mathbb{R}}^{\alpha}} \leq C\left\|\xi^{*}\right\|_{X^{*}}\|g\|_{\mathcal{M}_{X}^{\alpha}} .
$$

The lemma follows from this inequality. 
3. Vector-valued Hardy spaces. We start with the classical definition of a vectorvalued atom.

Definition 3.1. Let $0<p \leq 1$. A function $a \in L_{X}^{1}\left(\mathbb{R}^{n}\right)$ is called an $(X, p)$-atom, or simply an atom in $X$, if the following conditions hold:

(1) supp $a \subset Q$, where $Q$ is a cube on $\mathbb{R}^{n}$.

(2)

$$
\left(\frac{1}{|Q|} \int_{Q}\|a(x)\|_{X}^{2} d x\right)^{1 / 2} \leq|Q|^{-1 / p}
$$

(3) For every multi-index $\alpha$, with $|\alpha| \leq n(1 / p-1)$,

$$
\int_{\mathbb{R}^{n}} x^{\alpha} a(x) d x=0
$$

DEFINITION 3.2. A ( $p, \infty)$-atom in $X$, is a function $a$ satisfying (1), (3) above, and

$$
\|a\|_{L_{X}^{\infty}} \leq|Q|^{-1 / p}
$$

DefinItion 3.3. Given $0<p \leq 1$ as above, $H_{X}^{p}$ is the space of vector-valued distributions $f \in \mathscr{Y}_{S}^{\prime}\left(\mathbb{R}^{n}, X\right)$ such that $f=\sum_{i=1}^{\infty} \lambda_{i} a_{i}$, where $a_{i}$ is an atom in $X$ for every $i \in \mathbb{N}$ and $\sum_{i=1}^{\infty}\left|\lambda_{i}\right|^{p}<\infty$.

We define

$$
\|f\|_{H_{X}^{p}}=\inf \left(\sum_{i=1}^{\infty}\left|\lambda_{i}\right|^{p}\right)^{1 / p} .
$$

As usual

$$
d(f, g)=\|f-g\|_{H_{X}^{p}}^{p}
$$

defines an invariant metric in $H_{X}^{p}$.

REMARK 3.4. (1) Let $\varphi \in \mathscr{Y}\left(\mathbb{R}^{n}\right)$. If $a$ is an atom in $X$, then

$$
\int_{\mathbb{R}^{n}}\left(M_{\varphi}^{*}(a)(x)\right)^{p} d x \leq C_{\varphi},
$$

where $C$ does not depend on $a$.

(2) There exists a continuous seminorm $\rho$ in $\mathscr{Y}\left(\mathbb{R}^{n}\right)$ such that

$$
\left\|\int_{\mathbb{R}^{n}} a \varphi(x) d x\right\| \leq \rho(\varphi)
$$

for every atom $a$ in $X$ and $\varphi \in \mathscr{Y}\left(\mathbb{R}^{n}\right)$.

(3) If $a_{i}$ is an $(X, p)$-atom and $\sum_{i=1}^{\infty}\left|\lambda_{i}\right|^{p}<\infty$, then $\sum_{i=1}^{\infty} \lambda_{i} a_{i}$ converges in $\mathscr{Y}_{b}^{\prime}\left(\mathbb{R}^{n}, X\right)$, hence, the series always defines an element of $H_{X}^{p}$ and the convergence is in this space.

(4) $H_{X}^{p}$ is a complete metric space. 
The proof of (1) for $\varphi \geq 0$ radial and decreasing is the same as in [9, Theorem 4.3, page 275]. The general case follows from the fact that all the gauges $\left\|M_{\varphi}^{*}(f)\right\|_{p}$ are equivalent for $\varphi \in \mathscr{Y}\left(\mathbb{R}^{n}\right)$, with $\int_{\mathbb{R}^{n}} \varphi(x) d x \neq 0$. This fact is proved as in the scalar case (cf. [8]).

To prove (2), let $\varphi \in \mathscr{Y}\left(\mathbb{R}^{n}\right)$ and an atom $a$ in $X$. Then

$$
\left\|\int_{\mathbb{R}^{n}} a(x) \varphi(x) d x\right\|=\|a * \check{\varphi}(0)\| \leq M_{\check{\varphi}}^{*}(a)(y)
$$

for every $|y| \leq 1$, where $\breve{\varphi}(x)=\varphi(-x)$. Raising the inequality above to the $p$ th power and integrating over the unit ball, we obtain

$$
\left\|\int_{\mathbb{R}^{n}} a(x) \varphi(x) d x\right\| \leq C_{\varphi} .
$$

The Banach-Steinhaus theorem implies that the set of atoms defines an equicontinuous family in $\mathscr{S}^{\prime}\left(\mathbb{R}^{n}, X\right)$, which implies (2).

Statement (3) follows directly from (2).

That $H_{X}^{p}$ is complete follows from (3) taking a subsequence of a Cauchy sequence $\left\{f_{n}\right\}_{n=1}^{\infty}$ in $H_{X}^{p}$ satisfying

$$
\left\|f_{n_{k+1}}-f_{n_{k}}\right\|_{H_{X}^{p}}^{p}<\frac{1}{2^{k}}
$$

If we denote by $H_{\max }^{p}(X)$ the space of elements $f \in \mathscr{S}^{\prime}\left(\mathbb{R}^{n}, X\right)$ such that $M_{\varphi}^{*}(f) \in$ $L^{p}\left(\mathbb{R}^{n}\right), 0<p \leq 1$, then (1) in Remark 3.4 implies that we have a continuous inclusion $H_{X}^{p} \hookrightarrow H_{\max }^{p}(X)$. As in the scalar case, the space $H_{\max }^{p}(X)$ does not depends on the gauge $\left\|M_{\varphi}^{*}(f)\right\|_{p}$, where $\varphi \in \mathscr{Y}\left(\mathbb{R}^{n}\right)$, with $\int_{\mathbb{R}^{n}} \varphi(x) d x \neq 0$. Moreover, we can use the grand maximal function to define it. Latter's proof of the atomic decomposition of the scalar Hardy spaces can be adapted to prove that, every atom $a$ in $X$ can be decomposed as a series $a=\sum_{i=1}^{\infty} \lambda_{i} a_{i}$, where $a_{i}$ is a $(p, \infty)$-atom in $X$, with $\sum_{i=1}^{\infty}\left|\lambda_{i}\right|^{p}<C$, where $C$ is independent of $a$.

4. Duality. In this section, we state the duality between the vector-valued Hardy spaces and the space of Lipschitz vector measures with values in $X^{*}$. Then we prove that all these measures have densities that are Lipschitz continuous functions, hence we have the representation of the space $\left(H_{X}^{p}\right)^{*}$ that holds in the scalar case without any restriction on the Banach space $X$. Henceforth, we denote

$$
\alpha=n\left(\frac{1}{p}-1\right), \quad N=\left[n\left(\frac{1}{p}-1\right)\right] .
$$

The first step is to prove that the elements of the dual $\left(H_{X}^{p}\right)^{*}$ can be represented by measures $\mu \in \mathcal{M}_{X^{*}}^{\alpha}$ acting on atoms in $X$ by

$$
\int_{\mathbb{R}^{n}} a(x) d \mu
$$

Proposition 4.1. Let $0<p \leq 1$. For every $\Phi \in\left(H_{X}^{p}\right)^{*}$, there exists a measure $\mu \in$ $\mu_{X^{*}}^{\alpha}$ unique modulo polynomials of degree less than or equal to $N$ (measures with polynomial density), such that (4.2) holds for every atom in $X$. 
Proof. Denote by $L_{N}^{2}(Q, X)$ the space of functions $f \in L_{X}^{2}\left(\mathbb{R}^{n}\right)$, supported in $Q$ and with vanishing moments up to order $N$, and let

$$
\Theta_{N}(X)=\bigcup_{Q} L_{N}^{2}(Q, X) .
$$

If $f \in L_{N}^{2}(Q, X)$, the function $a(x)=|Q|^{1 / 2-1 / p}\|f\|_{L_{X}^{2}}^{-1} f(x)$ is an atom in $X$, then

$$
|\Phi(f)| \leq\|\Phi\|\|f\|_{L_{X}^{2}}|Q|^{1 / p-1 / 2} .
$$

We can extend this functional to the whole space $L_{X}^{2}(Q)$ without increasing the norm, and obtain a measure $\mu_{Q} \in V_{X^{*}}^{2}(Q)$, with $\left\|\mu_{Q}\right\|_{V_{X^{*}}^{2}(Q)} \leq\|\Phi\||Q|^{1 / p-1 / 2}$ such that

$$
\Phi(f)=\int_{Q} f d \mu_{Q}
$$

for every $f \in L_{N}^{2}(Q, X)$.

We show that $\mu_{Q}$ is uniquely determined up to addition of a measure with polynomial density with values in $X^{*}$.

Indeed, since $f \in L_{N}^{2}(Q, X)$ has vanishing moments up to order $N$, then for every polynomial $P$ of degree less than or equal to $N, \mu_{Q}+P \cdot m$ also satisfies (4.5). Conversely, suppose that $\mu_{1}$ and $\mu_{2}$ belong to $V_{X^{*}}^{2}(Q)$ and satisfy (4.5). Let $f \in L_{X}^{2}(Q), v=$ $\mu_{1}-\mu_{2}$ and consider the interpolation polynomials $P_{Q} f$ and $P_{Q} v$ of degree $N$. Then $f-P_{Q} f \in L_{N}^{2}(Q, X)$ and

$$
0=\int_{Q}\left(f-P_{Q} f\right) d v=\int_{Q}\left(f-P_{Q} f\right)\left(d v-P_{Q} v(x) d x\right)=\int_{Q} f\left(d v-P_{Q} v(x) d x\right)
$$

for every $f \in L_{X}^{2}(Q)$, therefore $d v=P_{Q} v(x) d x$.

We conclude in particular that there exists a unique measure $v_{Q} \in V_{X^{*}}^{2}(Q)$ satisfying (4.5) with vanishing moments of order less than or equal to $N$. This measure is

$$
v_{Q}=\mu_{Q}-\left(P_{Q} \mu_{Q}\right) m
$$

By Corollary 2.2, we have the estimate

$$
\left\|v_{Q}\right\|_{V_{X^{*}}^{2}(Q)} \leq C\|\Phi\||Q|^{1 / p-1 / 2} .
$$

Now, we decompose $\mathbb{R}^{n}=\cup_{j=1}^{\infty} Q_{j}$, where $\left(Q_{j}\right)$ is an increasing sequence of cubes. We can adjust the measures $\mu_{Q_{j}}$ adding measures with polynomial density of degree less than or equal to $N$ to obtain a single measure $\mu$ with values on $X^{*}$ and defined on $\sum_{0}$, such that

$$
\Phi(f)=\int_{\mathbb{R}^{n}} f d \mu
$$

for every $f \in \Theta_{N}(X)$. Since the restriction to every cube $Q$ of $\mu-\left(P_{Q} \mu\right) m$ is $\nu_{Q}$, then by (4.8) we conclude that $\mu \in \mathcal{M}_{X^{*}}^{\alpha}$ and

$$
\|\mu\|_{\mu_{X^{*}}^{\alpha}} \leq C\|\Phi\| .
$$


Since $\Theta_{N}(X)$ is dense in $H_{X}^{p}$, we have shown that

$$
\left(H_{X}^{p}\right)^{*} \longrightarrow \widetilde{M_{X^{*}}^{\alpha}} \text {. }
$$

Now, let $0<p<1$ and $\mu \in \mathcal{M}_{X^{*}}^{\alpha}$. We prove that the natural action of $\mu$ in $\Theta_{N}(X)$ given by

$$
\Phi(f)=\int_{\mathbb{R}^{n}} f d \mu
$$

can be extended to $H_{X}^{p}$ as a continuous functional.

Since

$$
\begin{aligned}
\left|\int_{Q} a(x) d \mu(x)\right| & =\left|\int_{Q} a(x) d\left(\mu-P_{Q}(\mu) m\right)\right| \\
& \leq\|a\|_{L_{X}^{2}}\left\|\mu-P_{Q}(\mu) m\right\|_{V_{X^{*}}^{2}(Q)} \leq\|\mu\|_{\mathcal{M}_{X^{*}}^{\alpha}},
\end{aligned}
$$

the proof of the continuity of $\Phi$ would be complete if we could prove that

$$
\Phi(f)=\sum \lambda_{j} \Phi\left(a_{j}\right),
$$

for every $f=\sum \lambda_{j} a_{j} \in \Theta_{N}(X)$ with atomic representation $f=\sum \lambda_{j} a_{j}$. The identity (4.14) holds if $\mu$ has a smooth density, as the following lemma shows.

LEMMA 4.2. Let $\psi \in \mathscr{S}\left(\mathbb{R}^{n}, X^{*}\right)$ and $f \in \Theta_{N}(X)$ with atomic representation $f=$ $\sum \lambda_{j} a_{j}$. Then

$$
\int_{\mathbb{R}^{n}}\langle f, \psi\rangle d x=\sum \lambda_{j} \int_{\mathbb{R}^{n}}\left\langle a_{j}, \psi\right\rangle d x
$$

(here $\langle$,$\rangle denotes the duality in X$ ).

Proof. Every atom $a$ in $X$ defines a continuous functional on $\mathscr{Y}\left(\mathbb{R}^{n}, X^{*}\right)$

$$
T_{a}(\psi)=\int_{\mathbb{R}^{n}}\langle a, \psi\rangle d x .
$$

Let $\rho$ be the continuous seminorm in $\mathscr{S}\left(\mathbb{R}^{n}\right)$ of Remark 3.4(2). Then for any $\psi=\sum \varphi_{i} \otimes$ $\xi_{i}^{*} \in \mathscr{Y}\left(\mathbb{R}^{n}\right) \otimes X^{*}$, we have

$$
\left|T_{a}(\psi)\right| \leq \sum \rho\left(\varphi_{i}\right)\left\|\xi_{i}^{*}\right\|_{X^{*}}
$$

Hence,

$$
\left|T_{a}(\psi)\right| \leq \tilde{\rho}(\psi)
$$

where $\tilde{\rho}$ is the continuous seminorm $\rho \otimes\|\cdot\|$ in the projective tensor product $\mathscr{Y}\left(\mathbb{R}^{n}\right) \otimes_{\pi}$ $X^{*}$. It follows that the family $\left\{T_{a}: a\right.$ is an atom in $\left.X\right\}$ is equicontinuous in $\left(\mathscr{Y}\left(\mathbb{R}^{n}\right) \hat{\otimes}_{\pi} X^{*}\right)^{\prime}=\mathscr{Y}\left(\mathbb{R}^{n}, X^{*}\right)^{\prime}$ (see $\left[12\right.$, Chapter 51]). We conclude that the series $\sum \lambda_{j} T_{a_{j}}$ converges in the strong topology of the dual space $\mathscr{Y}\left(\mathbb{R}^{n}, X^{*}\right)^{\prime}$ to a continuous functional. Since (4.15) holds for $\psi$ in the dense subset $\mathscr{Y}\left(\mathbb{R}^{n}\right) \otimes X^{*}$ of $\mathscr{Y}\left(\mathbb{R}^{n}, X^{*}\right)$, the proof of the lemma is now complete.

Now we are in position to prove the continuous inclusion $\mathcal{M}_{X}^{\alpha} \subset \Lambda_{X}^{\alpha}$. 
Proposition 4.3. Every $\mu \in M_{X}^{\alpha}$ has a density $g \in \Lambda_{X}^{\alpha}\left(\mathbb{R}^{n}\right)$ such that $\|g\|_{\Lambda_{X}^{\alpha}} \leq$ $C\|\mu\|_{\mu_{X}^{\alpha}}$.

Proof. Let $\varphi \in C_{c}^{\infty}$ radial, $\varphi \geq 0$, with supp $\varphi$ in the unit ball and such that $\int_{\mathbb{R}^{n}} \varphi=1$. Consider the family of measures $\left(\mu_{t}\right)_{t>0}$ with density $\varphi_{t} * \mu(x)=$ $\mu\left(\varphi_{t}(x-\cdot)\right)$, where $\varphi_{t}(x)=\left(1 / t^{n}\right) \varphi(x / t)$. Notice that $\mu_{t}$ is a $C^{\infty}$ function with values in $X$. In fact, for every $\xi^{*} \in X^{*}$, the measure $\xi^{*} \circ \mu$ has a Radon-Nikodym derivative in $L_{\text {loc }}^{1}$. Then $\xi^{*} \circ \mu_{t}=\left(\xi^{*} \circ \mu\right)_{t}$ is a smooth function, and this implies that $\mu_{t}$ is also smooth (see [11]).

By Lemma 2.4 we have

$$
\left\|\xi^{*} \circ \mu_{t}\right\|_{\Lambda^{\alpha}}=\left\|\left(\xi^{*} \circ \mu\right)_{t}\right\|_{\Lambda^{\alpha}} \leq C\left\|\left(\xi^{*} \circ \mu\right)_{t}\right\|_{\mathcal{M}_{\mathbb{R}}^{\alpha}},
$$

and [9, proof of Theorem 5.22, Chapter III]

$$
\left\|\left(\xi^{*} \circ \mu\right)_{t}\right\|_{\mathcal{M}_{\mathbb{R}}^{\alpha}} \leq C\left\|\xi^{*} \circ \mu\right\|_{\mathcal{M}_{\mathbb{R}}^{\alpha}} .
$$

But we have

$$
\left\|\xi^{*} \circ \mu\right\|_{\mathcal{M}_{\mathbb{R}}^{\alpha}} \leq\left\|\xi^{*}\right\|_{X^{*}}\|\mu\|_{\mathcal{M}_{X}^{\alpha}}
$$

and thus, we conclude that

$$
\left\|\mu_{t}\right\|_{\Lambda_{X}^{\alpha}} \leq C\|\mu\|_{\mu_{X}^{\alpha}} .
$$

We know that $\mu_{t}$ converges to $\mu$ in $\mathscr{D}^{\prime}\left(\mathbb{R}^{n}, X\right)$ as $t \rightarrow 0$. Then the proof of the proposition will be complete once we prove that $D^{\beta} \mu_{t}$ converges uniformly on compact sets of $\mathbb{R}^{n}$ for $|\beta| \leq[\alpha]$. To this end, recall that if $k$ is a nonnegative integer and $\beta$ is a multi-index such that $k+|\beta|>\alpha$. Then

$$
\frac{\partial^{k}}{\partial t^{k}} D_{x}^{\beta}\left(\varphi_{t}(x)\right)=C t^{\alpha-k-|\beta|} a_{t}(x), \quad x \in \mathbb{R}^{n}, t>0,
$$

where $a_{t}(x)$ is a scalar $(p, \infty)$-atom for every $t$, and $C$ is a constant depending on $\varphi$, $\alpha, n$, and the order of differentiation, but not on $t$ (see [9, Lemma 5.20, Chapter III]).

Then

$$
\begin{aligned}
\left\|\frac{\partial^{k}}{\partial t^{k}} D_{x}^{\beta} \mu_{t}(x, t)\right\|_{X} & =\left\|\int_{\mathbb{R}^{n}} \frac{\partial^{k}}{\partial t^{k}} D_{y}^{\beta}\left(\varphi_{t}(x-y)\right) d \mu(y)\right\|_{X} \\
& =C t^{\alpha-k-|\beta|}\left\|\int_{\mathbb{R}^{n}} a_{t}(x-y) d \mu(y)\right\|_{X}
\end{aligned}
$$

Proceeding as in (4.13), we see that

$$
\left\|\int_{\mathbb{R}^{n}} a_{t}(x-y) d \mu(y)\right\|_{X} \leq\|\mu\|_{\mathcal{M}_{X}^{\alpha}}
$$

Therefore, for any integer $k \geq 0$ and any multi-index $\beta$ such that $k+|\beta|>\alpha$,

$$
\left\|\frac{\partial^{k}}{\partial t^{k}} D_{x}^{\beta} \mu_{t}(x)\right\|_{X} \leq C t^{\alpha-k-|\beta|}\|\mu\|_{\mathcal{M}_{X}^{\alpha}}
$$

where $C$ does not depend on $t$ and $\mu$. 
With this inequality and the fundamental theorem of calculus it is easy to prove that $D_{x}^{\beta} \mu_{t}$ satisfies a uniform Cauchy estimate on compact sets on $\mathbb{R}^{n}$ as $t$ tends to zero. The proof is complete.

Now, we are ready to prove that

$$
\int_{\mathbb{R}^{n}} f d \mu=\sum \lambda_{j} \int a_{j} d \mu
$$

for every $f \in \Theta_{N}(X)$ with atomic representation $f=\sum \lambda_{j} a_{j}$ and every $\mu \in \mathcal{M}_{X^{*}}^{\alpha}$.

Let $g \in \Lambda_{X^{*}}^{\alpha}$ be the density of $\mu$. Let $h_{m}(x)=g(x) \psi(x / m)$, where $\psi$ is a radial and $C^{\infty}$ scalar function supported in the unit ball such that $\psi(x)=1$ for $|x| \leq 1 / 2$ and $0 \leq \psi(x) \leq 1$ for all $x$. The family $\left\{h_{m}\right\}_{m \in \mathbb{N}}$ is a sequence of functions in $\Lambda_{X^{*}}^{\alpha}$ with norm bounded by $C\|g\|_{\Lambda_{X^{*}}^{\alpha}}$. This cut-off process combined with the regularization described in the proof of Proposition 4.3 implies that there exists a sequence of functions $\left(g_{m}\right)_{m=1}^{\infty}$ in $C_{X^{*}}^{\infty}$ with compact support such that

(1) $\left\|g_{m}\right\|_{\Lambda_{X^{*}}^{\alpha}} \leq C\|g\|_{\Lambda_{X^{*}}^{\alpha}}$ for every $m \in \mathbb{N}$, where $C$ is an absolute constant,

(2) $\int\left\langle f, g_{m}\right\rangle d x \rightarrow \int\langle f, g\rangle d x$ as $m \rightarrow \infty$ for every $f \in \Theta_{N}(X)$.

Lemma 4.2 and the bounded convergence theorem imply (4.14). Thus, we have proved the following theorem.

THEOREM 4.4. Let $X$ be a Banach space over $\mathbb{R}$. For $0<p<1$ and $\alpha=n(1 / p-1)$,

$$
\left(H_{X}^{p}\right)^{*}=\tilde{\Lambda}_{X^{*}}^{\alpha}
$$

with equivalent norms.

ACKNOWLEDGEMENT. This work was partially supported by PAPIIT-UNAM IN1027 99 and CONACyT 32408-E.

\section{REFERENCES}

[1] O. Blasco, Hardy spaces of vector-valued functions: duality, Trans. Amer. Math. Soc. 308 (1988), no. 2, 495-507. MR 90a:46082. Zbl 658.46021.

[2] - On the dual space of $H_{B}^{1, \infty}$, Colloq. Math. 55 (1988), no. 2, 253-259. MR 89m:46068. Zbl 663.46028.

[3] __ Vector valued measures of bounded mean oscillation. Conference on Mathematical Analysis (El Escorial, 1989), Publ. Mat. 35 (1991), no. 1, 119-130. MR 92i:46051. Zbl 746.46040 .

[4] J. Bourgain, Vector-valued singular integrals and the $H^{1}-B M O$ duality, Probability Theory and Harmonic Analysis (Cleveland, Ohio, 1983), Monographs Textbooks Pure Appl. Math., vol. 98, Marcel Dekker, New York, 1986, pp. 1-19. MR 87j:42049b. Zbl 602.42015.

[5] A. V. Buhvalov, The conjugates of spaces of analytic vector-valued functions and the duality of functors generated by these spaces. Investigations on linear operators and the theory of functions, IX, Zap. Nauchn. Sem. Leningrad. Otdel. Mat. Inst. Steklov. (LOMI) 92 (1979), 30-50, 318 (Russian). MR 81b:46052. Zbl 431.46050.

[6] J. Diestel and J. J. Uhl, Jr., Vector Measures, Mathematical Surveys, no. 15, American Mathematical Society, Providence, R.I., 1977, with a foreword by B. J. Pettis. MR 56\#12216. Zbl 369.46039.

[7] N. Dinculeanu, Vector Measures, International Series of Monographs in Pure and Applied Mathematics, vol. 95, Pergamon Press, Oxford; VEB Deutscher Verlag der Wissenschaften, Berlin, 1967. MR 34\#6011b. 
[8] C. Fefferman and E. M. Stein, $H^{p}$ spaces of several variables, Acta Math. 129 (1972), no. 34, 137-193. MR 56\#6263. Zbl 257.46078.

[9] J. García-Cuerva and J. L. Rubio de Francia, Weighted Norm Inequalities and Related Topics, North-Holland Mathematics Studies, vol. 116, North-Holland Publishing Co., Amsterdam, 1985, Notas de Matematica [Mathematical Notes], vol. 104. MR 87d:42023. Zbl 578.46046.

[10] R. H. Latter, A characterization of $H^{p}\left(\mathbf{R}^{n}\right)$ in terms of atoms, Studia Math. 62 (1978), no. 1, 93-101. MR 58\#2198. Zbl 398.42017.

[11] L. Schwartz, Espaces de fonctions différentiables à valeurs vectorielles, J. Analyse Math. 4 (1954/55), 88-148 (French). MR 18,220a. Zbl 066.09601.

[12] F. Trèves, Topological Vector Spaces, Distributions and Kernels, Academic Press, New York, 1967. MR 37\#726. Zbl 171.10402.

MAGALI FolCh-GABAYET: INSTITUTO DE MATEMÁTICAS, UNIVERSIDAD NACIONAL AUTÓNOMA DE MÉXICO, CiUdAd Universitaria, D.F., 04510, MEXICO

E-mail address: folchgab@matem. unam. $\mathrm{mx}$

Martha GuZmán-Partida: Universidad de Sonora, Departamento de Matemáticas, BlvD. Luis EnCINAS y RoSALES, HeRMosillo, SONORA, 83000, MEXICO

E-mail address: martha@gauss . mat. uson. mx

SALVADOR PÉREZ-ESTEVA: INSTITUTO DE MATEMÁTICAS, UNIVERSIDAD NACIONAL AUTÓNOMA

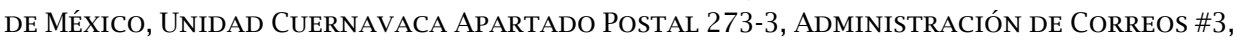
Cuernavaca, Morelos, 62251, MeXico

E-mail address: sa7vador@aluxe.matcuer.unam.mx 


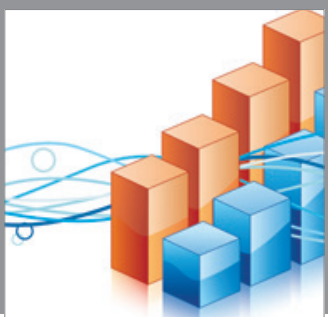

Advances in

Operations Research

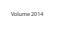

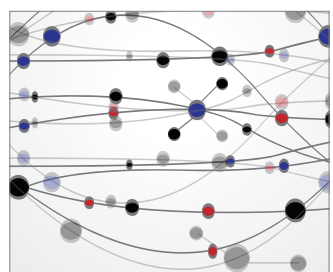

\section{The Scientific} World Journal
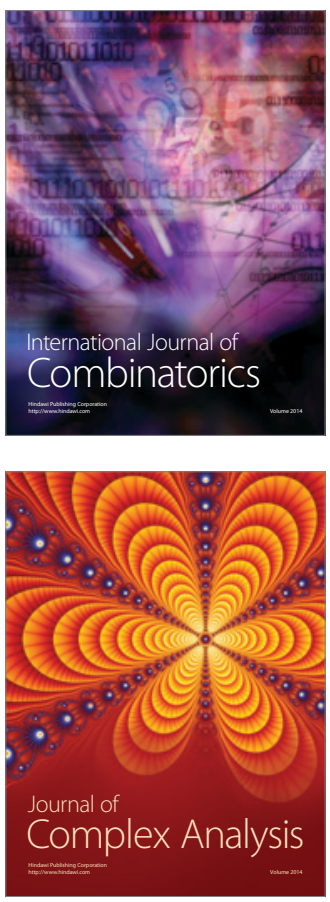

International Journal of

Mathematics and

Mathematical

Sciences
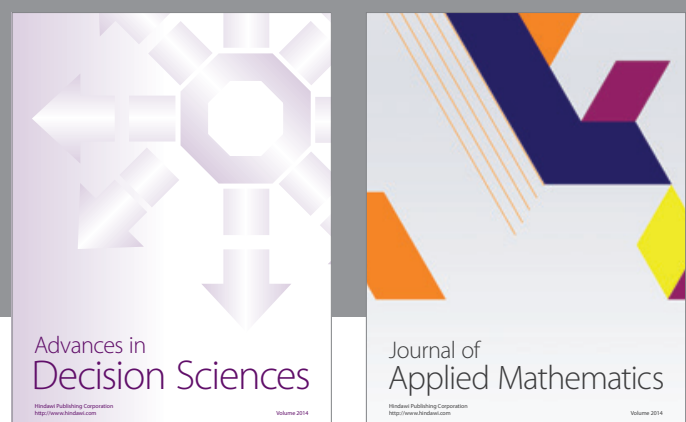

Journal of

Applied Mathematics
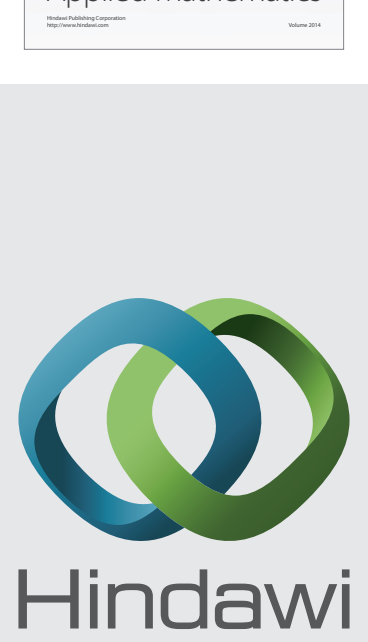

Submit your manuscripts at http://www.hindawi.com
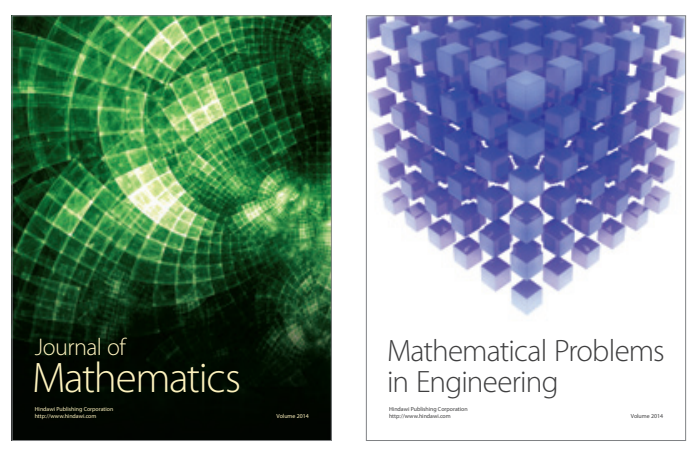

Mathematical Problems in Engineering
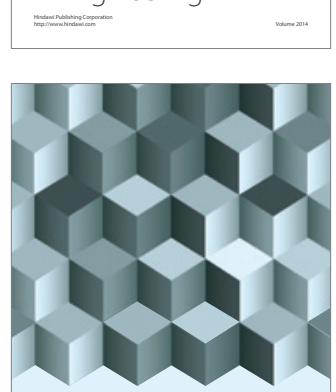

Journal of

Function Spaces
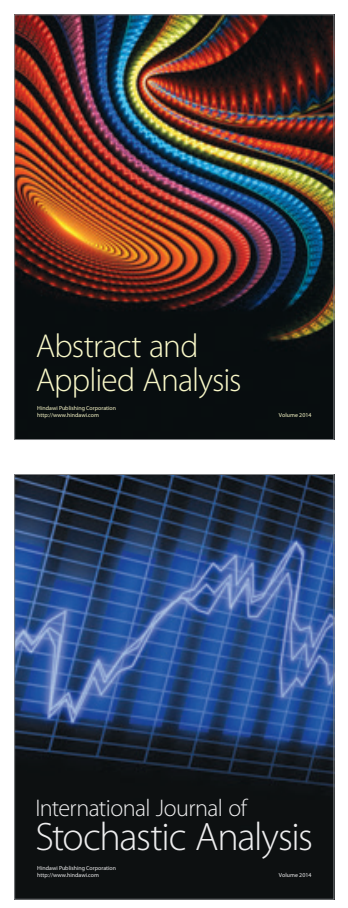

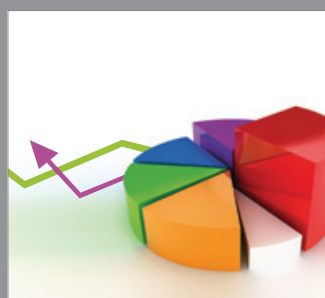

ournal of

Probability and Statistics

Promensencen
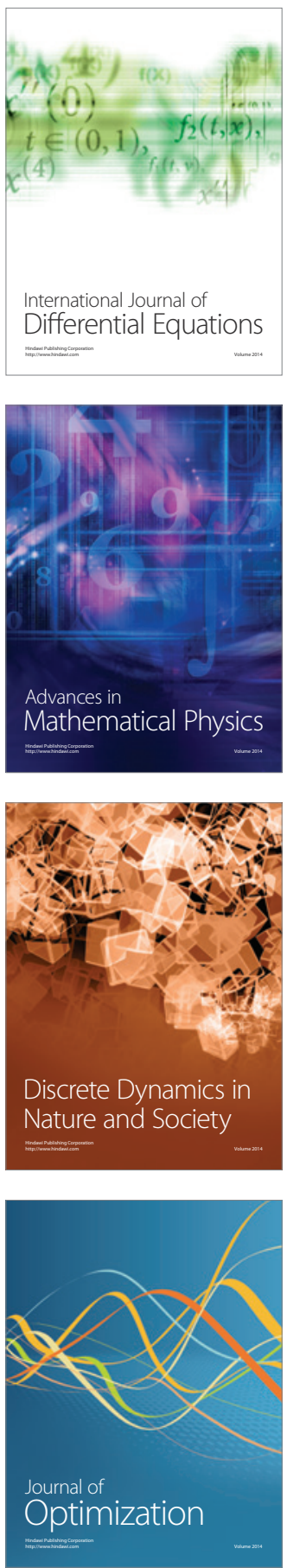